\title{
Studies of Ligand-Induced Site-Specific Phosphorylation of Epidermal Growth Factor Receptor
}

\author{
Lin Guo, Carl J. Kozlosky, Lowell H. Ericsson, Thomas O. Daniel, \\ Douglas P. Cerretti, and Richard S. Johnson \\ Amgen Corporation, Seattle, Washington, USA
}

The epidermal growth factor receptor (EGFR) is a receptor tyrosine kinase involved in the regulation of growth in many animal cells, including cancer cells. Phosphorylation of specific tyrosine residues within the cytoplasmic domain of EGFR is part of the initial activation process that occurs upon ligand binding, and these phosphotyrosine residues subsequently serve as docking sites for intracellular signaling molecules. To study the phosphorylation on each individual site, EGFR generated from a human epidermoid carcinoma cell line (A431) was analyzed by mass spectrometry. Liquid chromatography combined with tandem mass spectrometry (LC/MS/MS) was used to identify the tryptic phosphopeptides and their sites of phosphorylation (Y992, Y1045, Y1068, Y1086, S1142, Y1148, and Y1173). Ion intensities for the phosphorylated and unphosphorylated tryptic peptides containing the sites of phosphorylation were measured, and the intensity ratios were used to assess the degree of phosphorylation at each site. Ligand concentrations were varied for epidermal growth factor (EGF) and transforming growth factor alpha (TGF $\alpha$ ) as stimuli, and all of the EGFR tyrosine sites were consequently found to exhibit increased levels of phosphorylation, although at different rates and to different extents. Phosphorylation of Y992 appeared to plateau at lower concentrations of ligand, whereas the other sites continued to have increased phosphorylation throughout a wide range of concentrations. Only small differences could be detected between the EGF and the TGF $\alpha$-induced EGFR phosphorylation. Pretreatment of A431 cells with a small molecule EGFR inhibitor nearly eliminated the ligand-induced phosphorylation on all of the sites except for Y992 and Y1068. (J Am Soc Mass Spectrom 2003, 14, 1022-1031) (c) 2003 American Society for Mass Spectrometry

$\mathrm{M}$ ature EGFR is a type I membrane glycoprotein with 1186 amino acids consisting of an extracellular domain for ligand binding, a single membrane-spanning section, and a cytoplasmic protein kinase domain [1]. Like other proteins in the receptor tyrosine kinase family, EGFR play an important role in regulating cell functions such as proliferation, migration and differentiation. EGFR, also known as ErbB1 or HER1, is a member of the ErbB family of receptors, and can be activated by several different ligands-EGF, TGF $\alpha$, amphiregulin (AR), betacellulin (BTC), heparinbinding EGF (HB-EGF), and epiregulin (EPR) [2]. Although BTC, HB-EGF, and EPR can bind to both EGFR and ErbB4, there is no experimental evidence suggesting that EGF, TGF $\alpha$, and AR bind to receptors other than EGFR. Upon ligand binding, members of ErbB family form both homo- and hetero-dimers [3]. Dimer-

Published online July 21, 2003

Address reprint requests to Dr. R. S. Johnson, Amgen, 51 University Street, Seattle, WA 98101. E-mail: jsrichar@amgen.com ization activates the intrinsic protein tyrosine kinase activities of the receptors, leading to phosphorylation of specific tyrosine residues within the cytoplasmic domain [4-8]. These tyrosine phosphorylation sites serve as docking sites for intracellular signaling proteins containing Src homology 2 (SH2) or phosphotyrosine binding (PTB) domains [9] and provide a crucial connection between the external stimuli and internal signal transduction pathways.

A number of human tumors express high levels of EGFR and its ligands, and increased expression of EGFR has sometimes been found to correlate with poor prognosis for cancer patients [10]. Hence, EGFR has become a target for a number of oncology therapeutics under development. Recently, it has been reported that a small molecule EGFR-specific kinase inhibitor may affect a subset of patients, but evidence of significant responses in the treated patient population as a whole was less significant [11]. Since a key linkage between the external stimuli and internal signaling is tyrosine phosphorylation, a better understanding of the quanti- 
tative changes among the different phosphorylation sites in response to ligands and inhibitors may provide a better understanding of the mechanism of action of EGFR inhibition. Towards this end, a mass spectrometric study of EGFR phosphorylation was initiated.

Over a period of 15 years (1984-1999) of extensive study, six tyrosine residues from the carboxyl terminus of EGFR, distal from the tyrosine kinase domain (residue 688-955), were reported to be phosphorylated in vivo upon ligand stimulation. These include tyrosine residue 1068, 1148, 1173 [4], 1086 [5, 6], 992 [7], and 1045 [8]. These sites were mapped using in vivo ${ }^{32} \mathrm{P}$-labeling of truncated or point-mutated EGFR. During the past two decades, immunodetection of in vivo tyrosine phosphorylated EGFR after ligand activation has been the primary tool for researchers to monitor the extent of total phosphorylation within intact EGFR. However, a drawback of this approach is that it measures accumulative changes in overall phosphorylation, rather than dynamic changes at specific sites.

Methods involving radiological detection of ${ }^{32} \mathrm{P}$ labeled proteins, such as 2-D phosphopeptide analysis and solid phase Edman sequencing [12] are particularly useful for accounting for all of the ${ }^{32} \mathrm{P}$ originally incorporated into a protein. Nevertheless, for many laboratories the use of high levels of ${ }^{32} \mathrm{P}$ for in vivo labeling is not feasible, and non-radioactive methods are the only option. Immunodetection is often used; however, for monitoring specific sites this typically requires prior development of antibodies. Furthermore, stoichiometry is difficult to determine with this method. DNA mutagenesis has also been used to study phosphorylation; however, this tends to be mostly a hypothesis-driven approach rather than an unbiased screen.

Since the introduction of electrospray and matrixassisted laser desorption ionization [13, 14], mass spectrometry has emerged as the preferred non-radioactive method for characterizing protein phosphorylation. However, in a given sample it is usually very difficult to find the few spectra that represent phosphopeptides amongst all of the rest of the data. Towards this end various methods have been developed for the enrichment and identification of phosphopeptides [15-17]. Other means of locating and identifying phosphopeptides include comparisons with phosphatase treated controls [18], comparisons between positive and negative MALDI spectra [19], the use of specialized mass spectrometer scans $[20,21]$ or analyzing large numbers of MS/MS spectra of peptides generated by digesting a sample with several different proteases [22].

Most of the aforementioned mass spectrometric methods provide qualitative information, and there have been relatively few mass spectrometry methods described for phosphate quantitation. Isotope dilution methods could be used for this purpose and would likely be quite accurate; however, these methods require the synthesis of isotopically labeled peptides [23] or the use of isotope enriched cell culture media [24]. A few researchers have suggested that a comparison of mass spectrometric signals of phosphorylated peptides with internal controls (i.e., an unmodified peptide derived from the same protein) can provide adequate quantitation. The difference between these reported methods seems to be in the choice of internal standard, and whether it should be the corresponding unphosphorylated peptide $[25,26]$ or a peptide derived from the same protein that does not contain any modifications [27]. Ideally, one would want the internal standard to have physical and chemical properties that are most similar to that of the phosphorylated peptide to be quantitated. Assuming that phosphorylation does not influence proteolytic cleavage, the corresponding unphosphorylated peptide would be the best candidate. We chose to use the latter method to monitor gross changes in phosphorylation, and are subsequently using isotope labeled synthetic phosphopeptides [23] to verify the quantitation. In addition, pairs of unlabeled synthetic phosphorylated and unphosphorylated peptides were synthesized in order to gauge the effect of phosphorylation on ionization efficiency for individual peptides [27, 28].

A431 cells, a human epidermoid carcinoma cell line in which EGFR is over-expressed [29], were treated under various conditions, and EGFR was immunoprecipitated with an EGFR antibody. Each of the immunoprecipitates was subjected to one dimensional polyacrylamide gel electrophoresis, and the EGFR bands were excised and an in-gel trypsin digest was performed. Six previously known phosphorylation sites on EGFR were found in 5 tryptic peptides, as well as an additional unknown serine phosphorylation site. The site-specific changes in phosphorylation were monitored using LC-MS. EGFR phosphorylation following EGF or TGF $\alpha$ stimulation was compared, and the effect of an EGFR kinase inhibitor on ligand-induced phosphorylation was also examined.

\section{Experimental}

\section{Cell Culture}

Cultures of the human epidermoid carcinoma cell line A-431 [30] (American Type Culture Collection, Manassas, VA) were grown in Dulbecco's modified Eagle's medium containing $10 \%$ fetal bovine serum plus antibiotics until $80 \%$ confluence was reached. Cell culture continued for an additional $16 \mathrm{~h}$ in the absence of serum. Cells were either untreated or treated with ligands at the various concentrations (see the Results section) for $20 \mathrm{~min}$ at $37^{\circ} \mathrm{C}$. Approximately $1 \times 10 \mathrm{e} 7$ cells were used in each treatment. For experiments involving EGFR inhibition, cells were pretreated with AG1478 [4-(3-chloroanilino)-6,7-dimethoxyquinazoline] (LC Laboratories, Woburn, MA) 20 min prior to addition of $100 \mathrm{ng} / \mathrm{ml}$ of either EGF or TGF $\alpha$ (Upstate Biotechnology, Waltham, MA). 


\section{EGFR Immunoprecipitation}

After ligand treatment, cells were washed two times with ice-cold $150 \mathrm{mM} \mathrm{NaCl}, 100 \mathrm{mM}$ sodium phosphate $\mathrm{pH} 7.2$ (PBS) and the cells were lysed in PBS containing $1 \%$ Triton X-100, $1 \mathrm{mM} \mathrm{NaF}, 1 \mathrm{mM} \beta$-glycerol-phosphate, $1 \mathrm{mM}$ sodium orthovanadate, and a protease inhibitor cocktail (Roche Diagnostics Corporation, Indianapolis, IN). Cell lysates were precleared with Protein A-Sepharose and then immunoprecipitated with 4 $\mathrm{ug} / \mathrm{ml}$ anti-EGFR clone LA22 (Upstate Biotechnology).

\section{Protein Gel separation, Reduction, Alkylation, and Digestion}

Following immunoprecipitation, EGFR was isolated using SDS-PAGE under reducing conditions using Tris/glycine $4-20 \%$ gradient precast Novex gels (Invitrogen, Carlsbad, CA). Protein bands were stained using Colloidal Blue (Invitrogen). Gel bands were manually excised and the gel pieces were processed using an automated protein digestion station (Genomic Solutions, Ann Arbor, MI) that performed the tasks of reduction and alkylation of cysteines, tryptic digestion (Promega, Madison, WI), and extraction of the peptides.

\section{Mass Spectrometry}

Mass spectrometry analysis of tryptic peptides was performed on a Micromass QTOF 1 instrument (Manchester, United Kingdom). Peptides were sequenced by on-line microcapillary liquid chromatography-electrospray ionization-tandem mass spectrometry (LC/MS/MS) operated in a data-dependent mode, and phosphopeptides were identified using the Mascot program [31]. The capillary column was made from a piece of 50- $\mu \mathrm{m}$ i.d. fused silica line with an internal frit (New Objective, Cambridge, MA) that was packed with YMC ODS-AQ resin (Waters, Milford, MA). The LC gradient $(0-75 \%$ solvent $\mathrm{B}$ in $60 \mathrm{~min}$, solvent $\mathrm{A}=0.1 \%$ formic acid, $0.001 \%$ heptafluorobutyric acid (HFBA), and 5\% acetonitrile; solvent $\mathrm{B}=80 \%$ acetonitrile) was developed using an Eldex Micropro syringe pump (Napa, CA) operating at $8 \mu \mathrm{l} / \mathrm{min}$. A preinjector splitter cut the flow to approximately $250 \mathrm{nl} / \mathrm{min}$. The effluent of the column was directed into an Upchurch (Oak Harbor, WA) micro-tee containing a platinum electrode and a New Objective uncoated fused silica tip (360 $\mu$ m o.d., 20 $\mu \mathrm{m}$ i.d., pulled to a $10 \mu \mathrm{m}$ opening). Ion intensity data were obtained from mass spectra generated using microcapillary liquid chromatography-electrospray ionization-mass spectrometry (LC/MS).

\section{Peptide Synthesis}

Solid phase peptide synthesis was performed on an Applied Biosystems model 433A synthesizer using Fmoc chemistry with reagents and Fmoc-amino acids from Applied Biosystems (Foster City, CA). Fmoc-
Table 1. EFGR tryptic peptides containing phosphorylation sites

\begin{tabular}{ll}
\hline MW $^{\mathrm{a}}$ & \multicolumn{1}{c}{ Sequence } \\
\hline \hline 3606.57 & ALMDEEDMDDVVDADEY(992)LIPQQGFFSSPSTSR \\
3397.61 & Y(1045)SSDPTGALTEDSIDDTFLPVPEY(1068)INOSVPK \\
2398.23 & RPAGSVQNPVY(1086)HNOPLNPAPSR \\
2235.02 & GSHQIS(1142)LDNPDY(1148)QODFFPK \\
1209.56 & GSTAENAEY(1173)LR \\
\hline
\end{tabular}

aMonoisotopic molecular weights are calculated for the unphosphorylated peptides.

$\operatorname{Tyr}\left(\mathrm{PO}_{3} \mathrm{H}_{2}\right)-\mathrm{OH}$ was from NovaBiochem (San Diego, $\mathrm{CA}$ ) and stable-isotope labeled Fmoc- $\left[{ }^{15} \mathrm{~N}\right] \mathrm{Val}-\mathrm{OH}$ and Fmoc- $\left[{ }^{15} \mathrm{~N}\right] \mathrm{Ala}-\mathrm{OH}$ were from Bachem (Torance, CA).

Peptides were cleaved from the resin and deblocked using reagent $\mathrm{K}$ (trifluoroacetic acid, ethanedithiol and thioanisole). Resin was removed by filtration. Peptides were precipitated in cold methyl butyl ether followed by multiple washes with cold diethyl ether to remove deblocking reagents. Peptide purification was carried out on a semi preparative HPLC column, C18 $10 \times 250$ $\mathrm{mm}$ from Vydac (Hesperia, CA), using aqueous/acetonitrile gradients in $0.1 \%$ trifluoroacetic acid. Fractions of the major peak were collected, checked for purity by analytical HPLC and MALDI-MS. The pure fractions were pooled and lyophilized for storage.

\section{Results and Discussion}

\section{Identification of EGFR Phosphorylation Sites}

In order to monitor changes in ligand-induced EGFR phosphorylation, it was first necessary to find and characterize peptides that contained the various phosphorylation sites. In-gel tryptic digestion of immunoprecipitated EGFR was performed, and the tryptic peptides were analyzed by data-dependent LC/MS/ MS. To increase the likelihood of collecting MS/MS spectra of lower abundance precursor ions, consecutive LC/MS/MS acquisitions were performed over narrow precursor mass ranges (e.g., $100 \mathrm{u}$ wide precursor survey scans) [32]. In a few cases, precursor ion selection was used in order to obtain CID spectra to verify the structure of specific phosphopeptides (see below). All of the previously reported tyrosine phosphorylation sites (Table 1) were found, and their sequences were confirmed by tandem mass spectrometry (see below). In addition to tyrosine phosphorylation, a serine phosphorylation site (S1142) was identified.

Two of the tyrosine phosphorylation sites, Y1045 and Y1068, are located in the same tryptic peptide and the two monophosphorylated forms cannot be distinguished by mass alone. However, these two peptides could be separated by HPLC, and the MS/MS spectra clearly demonstrated their structural differences (Figure 1). Whereas the other sites had previously been identified on the basis of phosphopeptide mapping [4-7], ligand-induced phosphorylation at Y1045 was 


\section{(a) YSSDPTGALTEDSIDDTFLPVPEY*INQSVPK}

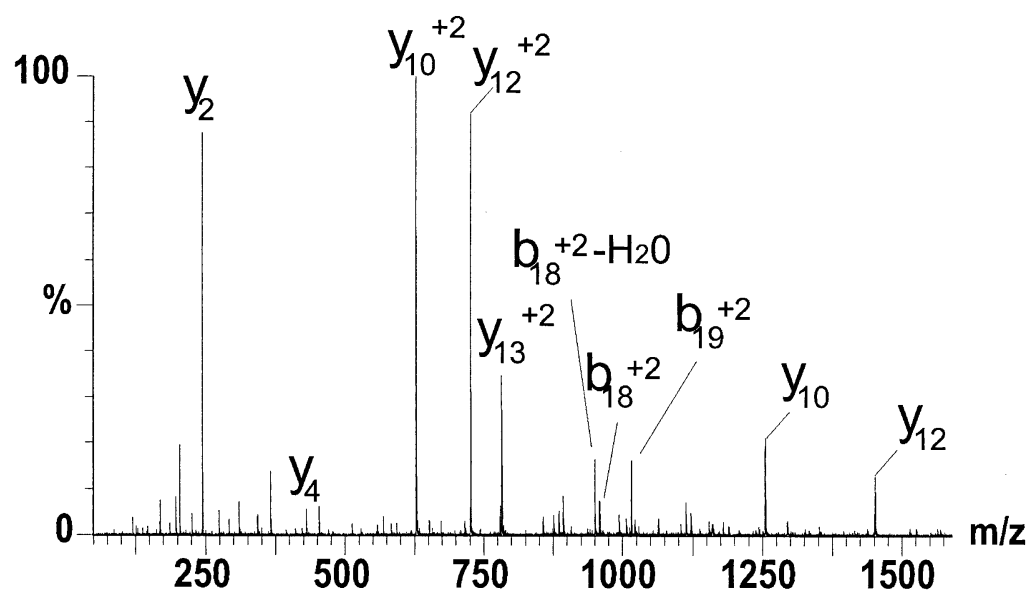

(b) $\quad Y^{*}$ SSDPTGALTEDSIDDTFLPVPEYINQSVPK

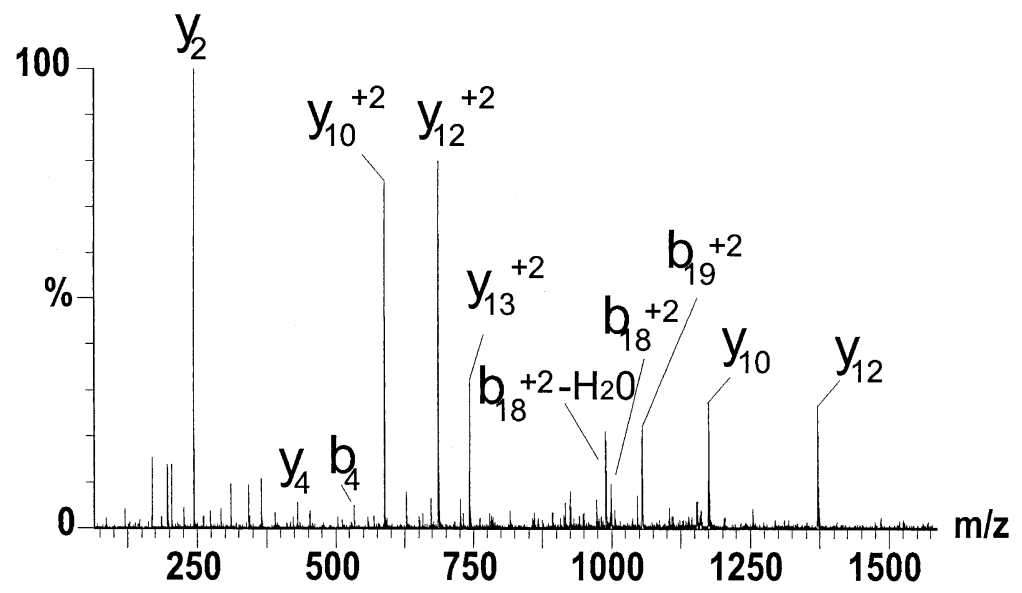

Figure 1. Tandem mass spectra of monophosphorylated EGFR tryptic peptides encompassing positions 1045-1075. (a) The earlier eluting peptide was identified as YSSDPTGALTEDSIDDTFLPVPEY*INQSVPK ( $Y^{*}$ represents phosphorylation at $\left.Y 1068\right)$. (b) The later eluting peptide was identified as $Y^{*}$ SSDPTGALTEDSIDDTFLPVPEYINQSVPK $\left(Y^{*}\right.$ represents phosphorylation at Y1045). The ion labeled $b_{4}$ is absent from the spectrum shown in panel (a). Ion assignments use the Biemann nomenclature [37].

implicated using site-directed mutagenesis data, and inhibitory effects of a synthetic phosphopeptide [8]. In contrast, the data reported here (Figure 1b) provides structural evidence that Y1045 is a ligand-induced phosphorylation site. The $b_{4}$ ion at $m / z 533.128$ indicates the presence of phosphate within the first three residues (YSS). The absence of an ion resulting from $\beta$-elimination of phosphate from the precursor ion, a fragmentation that is typical for peptides containing phosphorylated serine or threonine, suggests that the phosphate is located on tyrosine. The presence of a phosphorylation site at the N-terminus of this tryptic peptide (Y1045) could conceivably result in reduced efficiency of proteolytic cleavage; however, no evidence of a larger partial tryptic phosphopeptide encompassing this site was found. Another peptide containing two phosphor- ylation sites was found containing Y1148 and S1142, and the two monophosphorylated forms could be separated by HPLC and their structures were verified by MS/MS (Figure 2). For both peptides (Figure 1 and Figure 2) the doubly phosphorylated peptide ions were of very low abundance and their ion intensities could not be accurately measured.

Assessment of Quantitation Methods for Determining Changes in Ligand-Induced EGFR Phosphorylation

To analyze the changes in phosphorylation sites in a quantitative manner, the immunopurified EGFR has to accurately reflect the state of the protein in vivo. The 
(a)

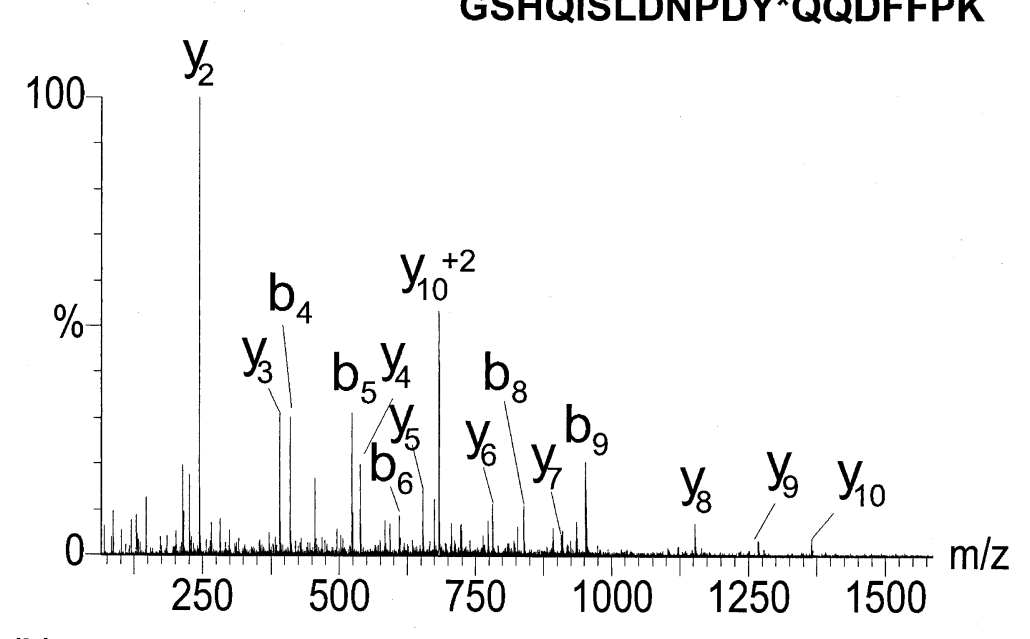

(b)

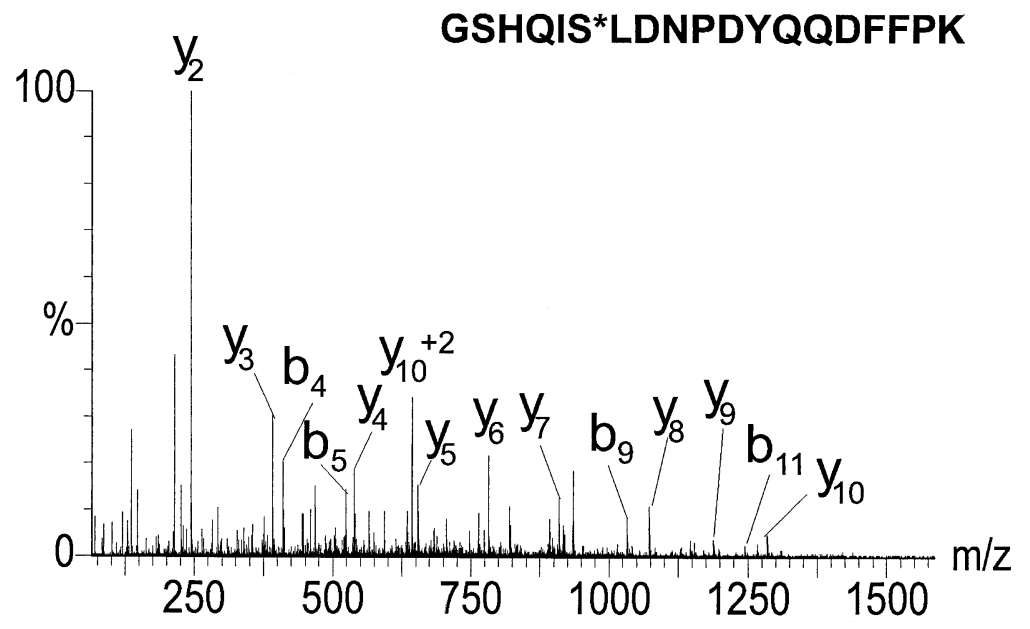

Figure 2. Tandem mass spectra of monophosphorylated EGFR tryptic peptides encompassing positions 1137-1154. (a) The earlier eluting peptide was identified as GSHQISLDNPDY*QQDFFPK (Y* represents phosphorylation at Y1148). (b) The later eluting peptide was identified as GSHQIS*LDNPDYQQDFFPK (S* represents phosphorylation at S1142). Some of the larger unlabeled peaks are b-type ions that had suffered a loss of phosphate via a gas phase beta-elimination reaction. Ion assignments use the Biemann nomenclature [37].

specific antibody that was used (LA22) [33] recognizes the extra cellular domain, and should therefore not be selective for or against different phosphorylation states of EGFR. Comparison of the Coomassie-stained EGFR bands from either unstimulated or EGF-stimulated cells showed that approximately the same amount of protein was recovered for each immunoprecipitation, despite the large differences found in the corresponding Western blots probed using an anti-phosphotyrosine antibody (data not shown).

Having characterized the observed tryptic EGFR phosphopeptides using LC/MS/MS (e.g., Figure 1 and Figure 2), a simpler analysis employing a single stage of mass spectrometry (i.e., LC/MS) is capable of monitoring changes in these phosphorylation sites under different stimulation conditions. Peptides in these LC/MS acquisitions are identified based on the $m / z$ value (with error less than $20 \mathrm{ppm}$ ), and by their chromatographic retention time. One of the difficulties in using mass spectrometry for quantitative analysis is that the fluctuation in instrument response needs to be normalized before different samples can be reliably compared. Ions derived from other EGFR tryptic peptides can serve this purpose, and we chose to use the ion intensity of the corresponding unphosphorylated tryptic peptide, since these are closely matched in terms of amino acid composition, mass, and HPLC elution time. Thus, simple ratios of the ion intensities of the phosphopeptides versus the corresponding unphosphorylated peptides were used to assess changes in phosphorylation for specific sites. The addition of phosphate to a peptide generally causes it to elute slightly earlier, depending on the peptide size. For example, the short 11 residue phospho-Y1173 peptide elutes nearly two min ahead of 
(a)

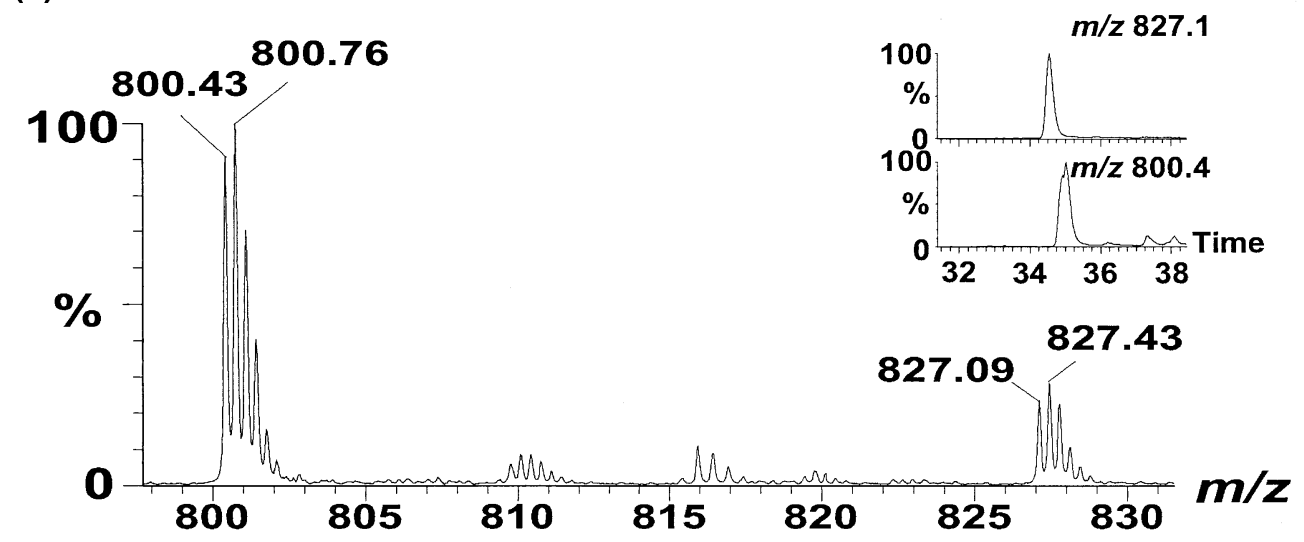

(b)

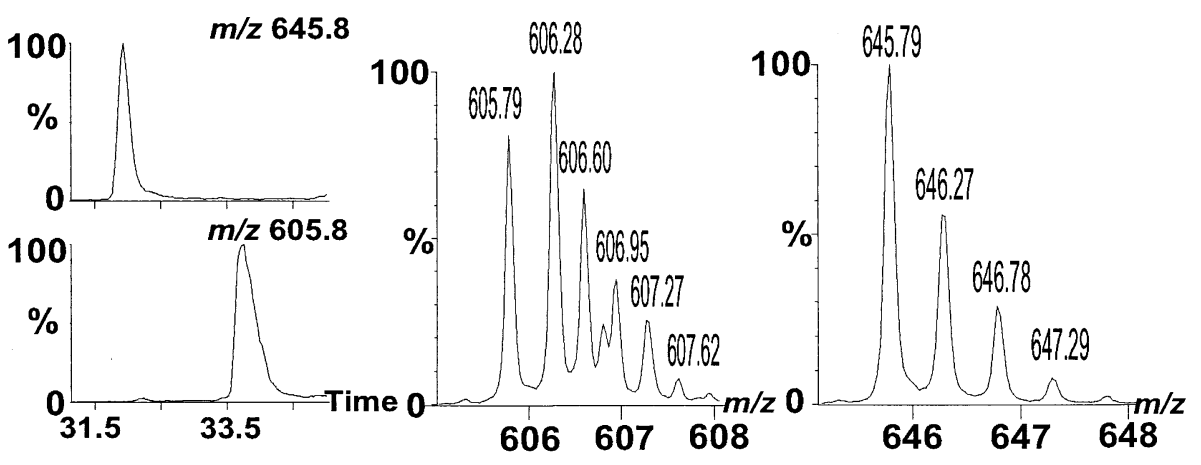

(c)

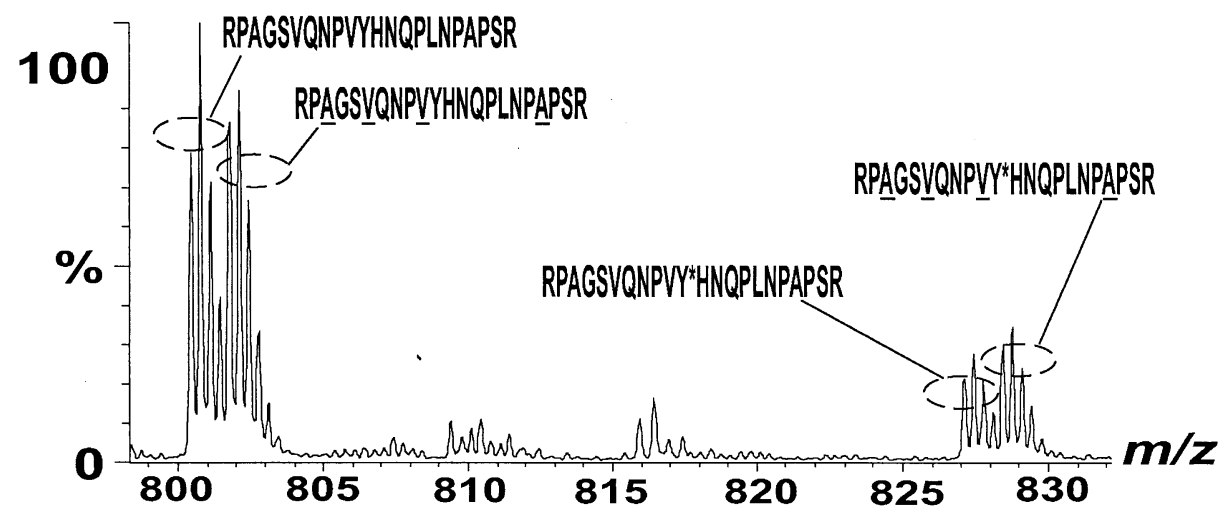

Figure 3. Site-specific quantitation of EGFR phosphorylation. (a) LC/MS mass spectrum (spectra were summed to include phosphorylated and unphosphorylated peptide ions) showing the triplycharged ions for the unphosphorylated $(\mathrm{m} / \mathrm{z}$ 800.43) and phosphorylated $(\mathrm{m} / \mathrm{z}$ 827.09) tryptic peptides containing Y1086-RPAGSVQNPVYHNQPLNPAPSR. The mass chromatograms for these two ions are shown in the insert. (b) LC/MS mass spectrum showing the doubly-charged ions for the unphosphorylated $(\mathrm{m} / \mathrm{z}$ 605.79) and phosphorylated $(\mathrm{m} / \mathrm{z}$ 645.79) tryptic peptides containing Y1173GSTAENAEYLR. The mass chromatograms for these two ions are shown on the left. (c) LC-MS spectrum obtained from a mixture of native and ${ }^{15} \mathrm{~N}$-labeled peptide RPAGSVQNPVYHNQPLNPAPSR in the phosphorylated and unphosphorylated forms, and the labeled amino acids are underlined.

the unphosphorylated peptide (Figure 3b, left), whereas most of the others behaved more like what is shown in the inset of Figure 3a. Usually the ions of interest were not contaminated by coeluting molecules with similar $\mathrm{m} / \mathrm{z}$ values, and quantitative data analysis in these cases was relatively simple (Figure 3a). However there were times when the data analysis was more complicated. For example, an unrelated triply-charged ion with the $\mathrm{m} / \mathrm{z}$ of 606.28 coeluted with the doubly-charged tryptic peptide containing $\mathrm{Y} 1173(\mathrm{~m} / \mathrm{z}$ of 605.79$)$ (Figure $3 \mathrm{~b}$, 
middle). By only using the intensity of the ${ }^{12} \mathrm{C}$ monoisotopic peak, ion intensity ratios of the phosphorylated and unphosphorylated peptide ions containing Y1173 could still be determined. Lower resolution instruments would not have been able to accurately distinguish the ion intensity contributions from the two peptides, thereby compromising the accurate determination of the ion intensity ratios.

Although changes in ion intensity ratios are indicative of changes in the level of phosphorylation, these ratios do not directly translate into stoichiometry. Peptide ion intensities vary according to the concentration of the peptides, suppression effects due to coeluting material, as well as peptide composition. It is also likely that addition of phosphate will have an impact on the ionization efficiency of a peptide [28]. To assess the effect of the addition of phosphate on ionization, three pairs of synthetic peptides were synthesized corresponding to the phosphorylated and unphosphorylated tryptic peptides containing Y1086, Y1148, and Y1173. Concentrated stock solutions of these synthetic peptides were made, and their concentrations were determined by amino acid analysis. From these stock solutions, a standard was prepared containing equimolar amounts of the phosphorylated and unphosphorylated peptides, and this standard mixture of synthetic peptides was analyzed using the same LC/MS conditions as was used to acquire data for the EGFR samples. Suprisingly, for these three pairs of peptides the ion intensity ratios were close to one $-\mathrm{pY} 1086 / \mathrm{Y} 1086=1.2$, pY1148 $/ \mathrm{Y} 1148$ $=0.9$, and $\mathrm{pY} 1173 / \mathrm{Y} 1173=1.1$. This indicated that for these three phosphorylation sites the ion intensity ratio of a peptide pair is, to some extent, a realistic assessment of the relative abundance of the peptides. It was noted, however, that this ratio was sensitive to the presence of the HPLC ion-pairing reagent HFBA and very different ratios were obtained in its absence. The presence of a very small amount of HFBA $(0.001 \%)$ was maintained throughout these experiments because of its beneficial effects on chromatographic resolution and retention of the more hydrophilic peptides.

Isotope dilution [34] is a standard method for the quantitation of specific compounds by mass spectrometry, and when applied to phosphorylation measurements it is possible to derive absolute quantitation [23]. This approach was feasible for some of the tryptic EGFR phosphopeptides, and peptides that incorporated ${ }^{15} \mathrm{~N}$ Val and ${ }^{15} \mathrm{~N}$-Ala were synthesized. The intent is to verify the quantitative measurements obtained using the reference peptide method. For example, standards corresponding to the phosphorylated and unphosphorylated tryptic peptide containing Y1086 (RPAGSVQNPVYHNQPLNPAPSR) were synthesized with the ${ }^{15} \mathrm{~N}$ label, which had masses that were $4 \mathrm{u}$ higher than the corresponding peptides obtained from the naturally occurring EGFR. By adding known quantities of the standards into EGFR samples following in-gel digestion, the absolute quantity of the pY1086 and Y1086 in the sample was determined (Figure 3c). It is recognized that inefficient extraction of the native peptides from the gel could result in an underestimation of the absolute quantities; however, assuming that the addition of phosphate would not affect the efficiency of extraction out of the gel, the relative molar ratios should be accurate. Differential losses could occur upon storage of the in-gel digest sample, but if the standards were added shortly after digestion, these losses would be matched by the standards.

\section{Concentration Studies of EGF and TGF $\alpha$ Induced EGFR Phosphorylation}

EGF and TGF $\alpha$ are homologous proteins that share similar three-dimensional structures, and are the two major ligands that activate EGFR. Although these two ligands exhibit similar biological effects, differences in downstream biological responses have been reported. How these two closely related ligands can elicit somewhat different biological signaling is an intriguing question. Like other ErbB receptor family members, downstream signaling by EGFR in response to different ligand stimulation can occur via different mechanisms [35] - ligand-induction can dictate the formation and activation of homo- or hetero-dimers, or differential phosphorylation of the receptor may send different signals.

To evaluate the contributions of EGFR phosphorylation on A431 cell ligand-induced downstream signal transduction, EGFR proteins were immunoprecipitated following EGF or TGF $\alpha$ stimulation of A431 cells using an antibody that recognizes the extracellular domain of EGFR. After further separation by gel electrophoresis, protein bands containing EGFR were cut out, and in-gel tryptic digestions were performed. For each predicated phosphorylation site, ion intensity information was gathered using LC/MS. The ion intensity ratios for each phosphorylated and unphosphorylated peptide pair upon ligand stimulation at a wide range of ligand concentrations were determined (Figure 4). Overall, EGF (Figure 4a) and TGF $\alpha$ (Figure 4b) induction yielded similar phosphorylation patterns at the different sites. A pair of isotope labeled synthetic phosphorylated and unphosphorylated peptides encompassing Y1086 were available, which were spiked into native EGF stimulated EGFR tryptic peptide samples. The results for this one phosphorylation site showed that the molar ratios measured using isotope dilution were nearly identical to the ion intensity ratio (data not shown).

Over a wide range of ligand stimulation $(0-1000$ $\mathrm{ng} / \mathrm{ml}$ ), all the phosphotyrosine sites showed increased phosphorylation, but to different degrees. For both ligands, the extent of Y992 phosphorylation seemed to plateau at a lower ligand concentration, whereas the other sites did not seem to reach a maximal level of phosphorylation even at $1000 \mathrm{ng} / \mathrm{ml}$ of ligand. Phosphorylation of Y1148 did not seem to occur to any 

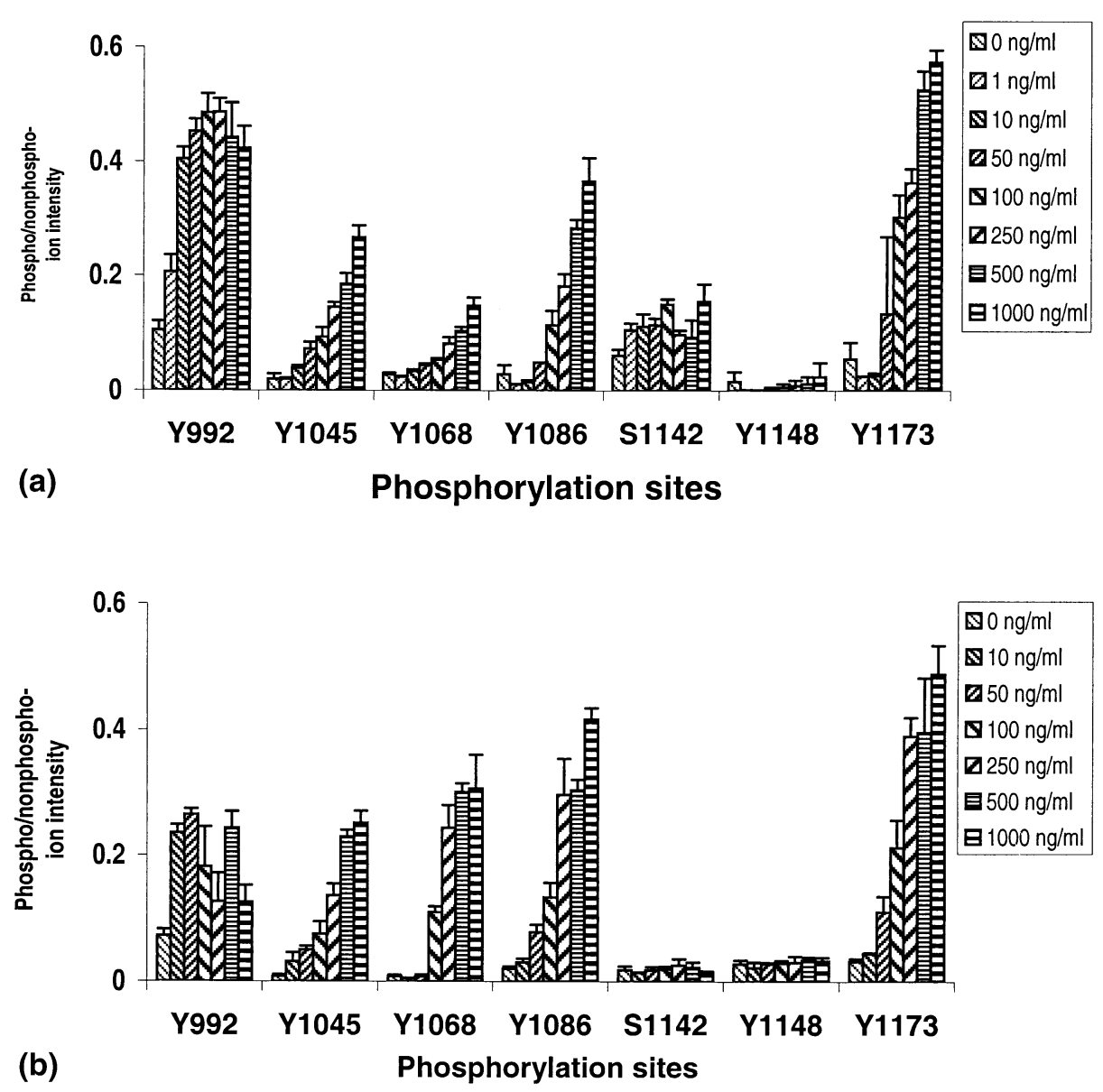

Figure 4. Ligand-induced changes in EGFR phosphorylation. (a) EGF-induced EGFR phosphorylation. (b) TGF $\alpha$-induced EGFR phosphorylation. A431 cells were cultured and stimulated with ligands at the indicated concentrations (see the method section). Data points are the average of three separate cell treatment samples, and the error bars show the standard deviation.

appreciable extent for either ligand. There was no clear effect on the phosphorylation of S1142, which is what would be expected for tyrosine kinase autophosphorylation.

\section{Effect of EGFR Inhibitors on Ligand-Induced Phosphorylation}

As shown above, different EGFR sites exhibit differences in the level of phosphorylation upon stimulation using varying concentrations of ligand. Our interest in this was to determine if these changes were of any relevance in the mechanism of action of EGFR inhibitors. Are there differences in how different sites respond to inhibitor treatment? Are some sites more sensitive to inhibition than others? To address these questions, AG1478, a highly potent inhibitor of EGF receptor kinase [36], was used to treat A431 cells prior to EGF stimulation. Mass spectrometric analysis of the EGFR phosphorylation sites indicated that four of the phosphorylation sites, Y1045, Y1086, Y1148, and Y1173, are very sensitive to inhibitor treatment, whereas Y992 and Y1068 are still phosphorylated at a significant level even at the highest concentration of inhibitor (Figure 5). The inhibitor had no effect on the level of phosphorylation of S1142, as expected.

\section{Conclusions}

After stimulation with EGF or TGF $\alpha$, LC/MS/MS was used to show that all of the known tyrosine phosphorylation sites, plus a previously uncharacterized serine phosphorylation site, were located within five tryptic peptides generated by in-gel digestion of immunoprecipitated EGFR. This formed the basis of an assay for analyzing the changes in phosphorylation. Changes in phosphorylation were monitored by comparison of the ion intensity of phosphopeptides with the unmodified peptide that was also derived from EGFR. Using this technique we were able to identify those sites that were most responsive to stimulation, and compare the sitespecific response obtained using two different ligands (EGF and TGF $\alpha$ ). In addition, we were able to monitor the effects of an EGFR kinase inhibitor on the phosphorylation state of these specific sites. This analysis has allowed us to investigate more precisely, the mecha- 


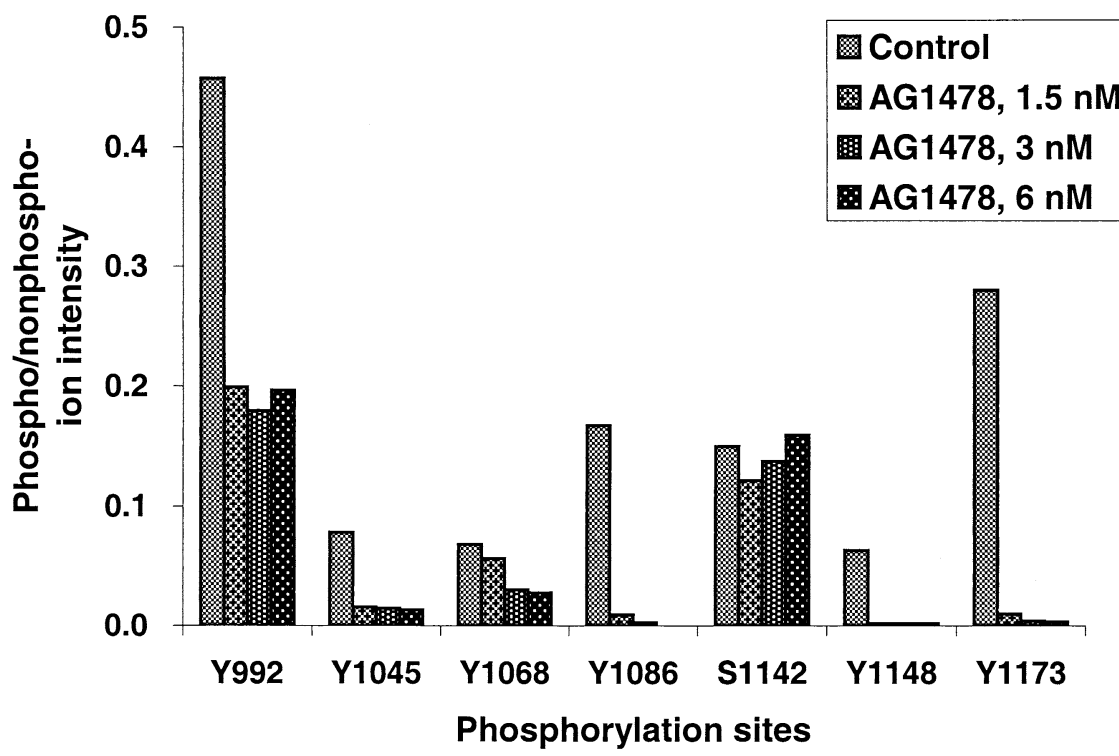

Figure 5. Effects of EGFR kinase inhibitor AG1478 on EGF-induced EGFR phosphorylations. The A431 cells were treated with the inhibitor for $20 \mathrm{~min}$ prior to the addition of $100 \mathrm{ng} / \mathrm{ml}$ EGF.

nism of action of the EGFR and has wide applications to similar protein such as other RTKs, cytoplasmic kinases, and phosphatases. These methods may also be useful in the clinical arena where extensive efforts are under way to test the therapeutic potential of drugs that modulate the enzymatic activity of kinases.

\section{References}

1. Carpenter, G. Receptors for Epidermal Growth Factor and Other Polypeptide Mitogens. Annu. Rev. Biochem. 1987, 56, 881-914.

2. Olayioye, M. A.; Neve, R. M.; Lane, H. A.; Hynes, N. E. The ErbB Signaling Network: Receptor Heterodimerization in Development and Cancer. Embo. J. 2000, 19, 3159-3167.

3. Boni-Schnetzler, M.; Pilch, P. F. Mechanism of Epidermal Growth Factor Receptor Autophosphorylation and HighAffinity Binding. Proc. Natl. Acad. Sci. U.S.A. 1987, 84, 78327836.

4. Downward, J.; Parker, P.; Waterfield, M. D. Autophosphorylation Sites on the Epidermal Growth Factor Receptor. Nature 1984, 311, 483-485.

5. Hsuan, J. J.; Totty, N.; Waterfield, M. D. Identification of a Novel Autophosphorylation Site (P4) on the Epidermal Growth Factor Receptor. Biochem. J. 1989, 262, 659-663.

6. Margolis, B. L.; Lax, I.; Kris, R.; Dombalagian, M.; Honegger, A. M.; Howk, R.; Givol, D.; Ullrich, A.; Schlessinger, J. All Autophosphorylation Sites of Epidermal Growth Factor (EGF) Receptor and HER2/neu are Located in Their CarboxylTerminal Tails. Identification of a Novel Site in EGF Receptor. J. Biol. Chem. 1989, 264, 10667-10671.

7. Walton, G. M.; Chen, W. S.; Rosenfeld, M. G.; Gill, G. N. Analysis of Deletions of the Carboxyl Terminus of the Epidermal Growth Factor Receptor Reveals Self-Phosphorylation at Tyrosine 992 and Enhanced in Vivo Tyrosine Phosphorylation of Cell Substrates. J. Biol. Chem. 1990, 265, 1750-1754.

8. Levkowitz, G.; Waterman, H.; Ettenberg, S. A.; Katz, M.; Tsygankov, A. Y.; Alroy, I.; Lavi, S.; Iwai, K.; Reiss, Y.; Ciechanover, A.; Lipkowitz, S.; Yarden, Y. Ubiquitin Ligase Activity and Tyrosine Phosphorylation Underlie Suppression of Growth Factor Signaling by c-Cbl/Sli-1. Mol. Cell 1999, 4, 1029-1040.

9. Shoelson, S. E. SH2 and PTB Domain Interactions in Tyrosine Kinase Signal Transduction. Curr. Opin. Chem. Biol. 1997, 1, 227-234.

10. Herbst, R. S.; Shin, D. M. Monoclonal Antibodies to Target Epidermal Growth Factor Receptor-Positive Tumors: A New Paradigm for Cancer Therapy. Cancer 2002, 94, 1593-1611.

11. Couzin, J. Cancer Drugs. Smart Weapons Prove Tough to Design. Science 2002, 298, 522-525.

12. Campbell, D.; Morrice, N. Identification of Protein Phosphorylation Sites by a Combination of Mass Spectrometry and Solid Phase Edman Sequencing. J. Biomol. Tech. 2002, 13, 119-130.

13. Fenn, J. B.; Mann, M.; Meng, C. K.; Wong, S. F.; Whitehouse, C. M. Electrospray Ionization for Mass Spectrometry of Large Biomolecules. Science 1989, 246, 64-71.

14. Karas, M.; Hillenkamp, F. Laser Desorption Ionization of Proteins with Molecular Masses Exceeding 10,000 Daltons. Anal. Chem. 1988, 60, 2299-2301.

15. Ficarro, S. B.; McCleland, M. L.; Stukenberg, P. T.; Burke, D. J.; Ross, M. M.; Shabanowitz, J.; Hunt, D. F.; White, F. M. Phosphoproteome Analysis by Mass Spectrometry and Its Application to Saccharomyces cerevisiae. Nat. Biotechnol. 2002, 20, 301-305.

16. Oda, Y.; Nagasu, T.; Chait, B. T. Enrichment Analysis of Phosphorylated Proteins as a Tool for Probing the Phosphoproteome. Nat. Biotechnol. 2001, 19, 379-382.

17. Zhou, H.; Watts, J. D.; Aebersold, R. A Systematic Approach to the Analysis of Protein Phosphorylation. Nat. Biotechnol. 2001, 19, 375-378.

18. Zhang, X.; Herring, C. J.; Romano, P. R.; Szczepanowska, J.; Brzeska, H.; Hinnebusch, A. G.; Qin, J. Identification of Phosphorylation Sites in Proteins Separated by Polyacrylamide Gel Electrophoresis. Anal. Chem. 1998, 70, 2050-2059.

19. Ma, Y.; Lu, Y.; Zeng, H.; Ron, D.; Mo, W.; Neubert, T. A. Characterization of Phosphopeptides from Protein Digests Using Matrix-Assisted Laser Desorption/Ionization Time-ofFlight Mass Spectrometry and Nanoelectrospray Quadrupole Time-of-Flight Mass Spectrometry. Rapid Commun. Mass Spectrom. 2001, 15, 1693-1700. 
20. Zappacosta, F.; Huddleston, M. J.; Karcher, R. L.; Gelfand, V. I.; Carr, S. A.; Annan, R. S. Improved Sensitivity for Phosphopeptide Mapping Using Capillary Column HPLC and Microionspray Mass Spectrometry: Comparative Phosphorylation Site Mapping from Gel-Derived Proteins. Anal. Chem. 2002, 74, 3221-3231.

21. Steen, H.; Kuster, B.; Fernandez, M.; Pandey, A.; Mann, M. Detection of Tyrosine Phosphorylated Peptides by Precursor Ion Scanning Quadrupole TOF Mass Spectrometry in Positive Ion Mode. Anal. Chem. 2001, 73, 1440-1448.

22. MacCoss, M. J.; McDonald, W. H.; Saraf, A.; Sadygov, R.; Clark, J. M.; Tasto, J. J.; Gould, K. L.; Wolters, D.; Washburn, M.; Weiss, A.; Clark, J. I.; Yates, J. R., III. Shotgun Identification of Protein Modifications from Protein Complexes and Lens Tissue. Proc. Natl. Acad. Sci. U.S.A. 2002, 99, 7900-7905.

23. Stemmann, O.; Zou, H.; Gerber, S. A.; Gygi, S. P.; Kirschner, M. W. Dual Inhibition of Sister Chromatid Separation at Metaphase. Cell 2001, 107, 715-726.

24. Oda, Y.; Huang, K.; Cross, F. R.; Cowburn, D.; Chait, B. T. Accurate Quantitation of Protein Expression and Site-Specific Phosphorylation. Proc. Natl. Acad. Sci. U.S.A. 1999, 96, 65916596.

25. Tsay, Y. G.; Wang, Y. H.; Chiu, C. M.; Shen, B. J.; Lee, S. C. A Strategy for Identification and Quantitation of Phosphopeptides by Liquid Chromatography/Tandem Mass Spectrometry. Anal. Biochem. 2000, 287, 55-64.

26. Asara, J. Lane, W. Ratio Analyzed Quantitation (RAQ) of Phosphorylation Sites from Different Protein States by LC/ MS/MS. Proceedings of the 50th Conference on Mass Spectrometry and Allied Topics; Orlando, FL, 2002.

27. Ruse, C. I.; Willard, B.; Jin, J. P.; Haas, T.; Kinter, M.; Bond, M. Quantitative Dynamics of Site-Specific Protein Phosphorylation Determined Using Liquid Chromatography Electrospray Ionization Mass Spectrometry. Anal. Chem. 2002, 74, 16581664 .
28. Lee, K. A.; Craven, K. B.; Niemi, G. A.; Hurley, J. B. Mass Spectrometric Analysis of the Kinetics of in Vivo Rhodopsin Phosphorylation. Protein Sci 2002, 11, 862-874.

29. Wrann, M. M.; Fox, C. F. Identification of Epidermal Growth Factor Receptors in a Hyperproducing Human Epidermoid Carcinoma Cell Line. J. Biol. Chem. 1979, 254, 8083-8086.

30. Giard, D. J.; Aaronson, S. A.; Todaro, G. J.; Arnstein, P.; Kersey, J. H.; Dosik, H.; Parks, W. P. In Vitro Cultivation of Human Tumors: Establishment of Cell Lines Derived from a Series of Solid Tumors. J. Natl. Cancer Inst. 1973, 51, 1417-1423.

31. Perkins, D. N.; Pappin, D. J.; Creasy, D. M.; Cottrell, J. S. Probability-Based Protein Identification by Searching Sequence Databases. Electrophoresis 1999, 20, 3551-3567.

32. Spahr, C.; Susin, S.; Bures, E.; Robinson, J.; Davis, M.; McGinley, M.; Kroemer, G.; Patterson, S. Simplification of Complex Peptide Mixtures for Proteomic Analysis: Reversible Biotinylation of Cysteinyl Peptides. Electrophoresis 2000, 21, 16351650.

33. Wu, D. G.; Wang, L. H.; Sato, G. H.; West, K. A.; Harris, W. R.; Crabb, J. W.; Sato, J. D. Human Epidermal Growth Factor (EGF) Receptor Sequence Recognized by EGF Competitive Monoclonal Antibodies. Evidence for the Localization of the EGF-Binding Site. J. Biol. Chem. 1989, 264, 17469-17475.

34. Chapman, J. R. Practical Organic Mass Spectrometry; John Wiley and Sons, Ltd.: West Sussex, 1993; pp. 272-305.

35. Sweeney, C; Carraway, K. L., III. Ligand Discrimination by ErbB Receptors: Differential Signaling Through Differential Phosphorylation Site Usage. Oncogene 2000, 19, 5568-5573.

36. Gazit, A.; Chen, J.; App, H.; McMahon, G.; Hirth, P.; Chen, I.; Levitzki, A. Tyrphostins IV-Highly Potent Inhibitors of EGF Receptor Kinase. Structure-Activity Relationship Study of 4-Anilidoquinazolines. Bioorg. Med. Chem. 1996, 4, 1203-1207.

37. Biemann, K. Appendix V. Nomenclature for Peptide Fragment Ions (Positive Ions). Methods Enzymol 1990, 193, 886-887. 